\title{
Extensive Methodologies for Design of User Experience by Systematic Innovations
}

\author{
Song-Kyoo Kim
}

\begin{abstract}
The paper deals Systematic Innovation method adaptations for revealing the universal framework design of user experience enhancement. User Experience is the way a person feels about using a product, system or services. The key idea of the enhanced user experience is behavior analysis based on the typical statistical data that can be gathering during the regular user operations. The proposed new user interface enhancing framework is targeted to give general guidelines of simpler user experiences design.
\end{abstract}

Index Terms-Behavior analysis, user experiences, UX design, TRIZ, TIPS, innovation, systematic innovation.

\section{INTRODUCTION}

User Experience (UX) is the way a person feels about using a product, system or service and it is subjective in nature, because it is also about feelings of individuals and thoughts about the system. User experience is dynamic, because it changes over time as the circumstances change. It highlights the experiential, affective, meaningful and valuable aspects of human-computer interaction and product ownership, but it also includes a person's perceptions of the practical aspects such as utility, ease of use and efficiency of the system.

Technically speaking, workflows and steps for exciting a function is major part of the UX. Even though, UX considers human feeling and values, interactions, it can be represented by technical (or algorithmically) explanation that makes adapting the human feelings into the machines. Consistency and simplicity are the core concept of User Experience design. Consistency is DNA (Deoxyribonucleic Acid) of the UX for the consumer electronics. It makes more strong appeals to customers who have used the products before and make it easy for transferring a new product. If the products do not have UX consistency, the products can not survive more than one product life cycle (12 months to 18 months for mobile phone devices). Maintaining the simple UX is more crucial part when the new functions are added. Usually, the UX is getting complicated when the functions are added.

Behavioral models typically integrate insights from psychology with neo-classical economic theory. It covers a range of concepts, methods, and fields. Behavioral Economics and Behavioral Finance are closely related fields making up a separate branch of economic and financial analysis.

This field uses social, cognitive and emotional factors in

Manuscript received November 16, 2012; revised January 16, 2013.

Song-Kyoo Kim is with the IEDRC and Asian Institute of Management, Makati City 1260, Philippines (e-mail: SKim@aim.edu). understanding the economic decisions of consumers, borrowers and investors, and their effects on market prices, returns and the allocation of resources. Behavioral analysts are not only concerned with the effects of market decisions but also with public choice, which describes another source of economic decisions with related biases towards promoting self-interest.

The key idea of the enhanced user experience is behavior analysis based on the typical statistical data that can be gathering during the regular user operations. User Experiences on consumer electronics such as a MP3 player, a mobile phone are considered as the target to apply the enhancement based on Systematic Innovation method because current consumer electronics are getting complicated and hard to learn the user interfaces (UI).

Currently, consumer electronics are powerful enough to support for improving the life style. For instant, a smartphone is basically a phone but also contain more than thousands of songs, play the full length of movies and do web browsing almost same as what is currently available on PC. Portability is one of key properties for most of consumer electronics but UI is limited because of this property. There are several approaches to improve the user experiences and some of companies such as Apple and Nintendo have shown to markets for innovative ways to improving user experiences dramatically. Human behavior in behavioral economics [1]-[3] is considered for innovative user experience enhancement. Behavioral economics is a separate branch of economics that is using social, cognitive and emotional factors in understanding the economic decisions of consumers. Even though the topic is relative new area in economics, it is popular subject and has been announced to public as a famous best seller authored by Thaler [2].

\section{GeNERAL FramewORK FOR BeHAVIOR BASED USER EXPERIENCE DESIGN}

Ideal final result (IFR) is a description of the best possible solution for the problem situation (or contradiction), regardless of the resources or constraints of the original problem. IFR is one of the basics terms in TRIZ (TIPS; Theory of Inventive Problem Solving) that is one of famous problem solving tool of Systematic Innovation method [4]-[8].

A well-defined IFR helps a problem solver to overcome psychological inertia and reach breakthrough solutions by thinking about the solution in terms of functions, not the intervening problems or needed resources. It focuses on functions needed, not the current process or equipment. It is therefore the antithesis to the more commonly used 
continuous improvement method which often leads to progressively diminishing returns (the classic S-curve). IFR represents a significant shift in the thinking approach to solving problems.

$$
\text { Ideality }=\frac{\sum_{i}^{\infty} U_{i}}{\sum_{j}^{\infty} H_{j}}
$$

The concept design of UX based on Su-Field model can be determined couple for solutions within 76 Inventive Standard solutions. In Amang's notation [9], the concept problems for the effective UX are either Type-1 or Type-2 problems:

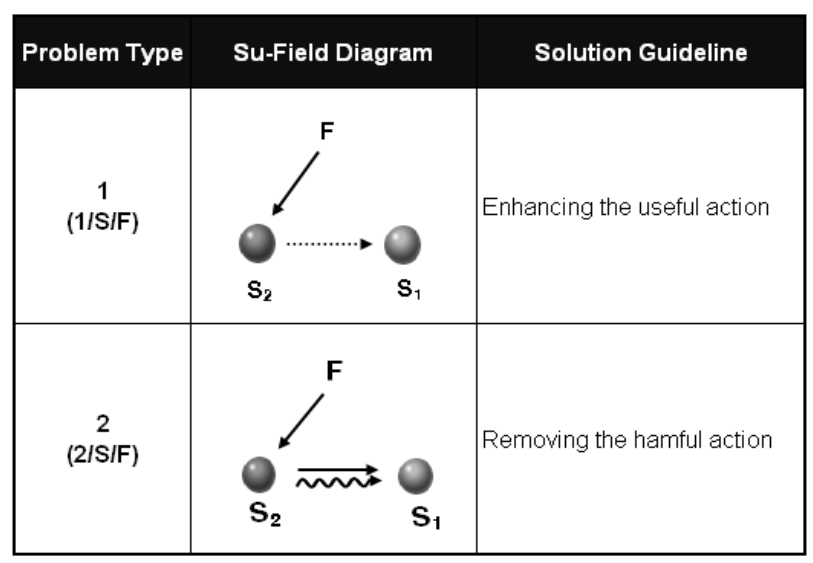

Fig. 1. Problem types in amang notation.

The notation method is clarifying the Inventive Standards simpler ways and users can be guided to the candidate solutions from the problems based on Su-Field model with the minimal knowledge of 76 Inventive Standard solutions. The new notation for Su-Field model (Su-Field notation) is provided [9]. The Su-Field model for Inventive standard solution can exhibits the summarized main characteristics of a Su-Field model.

$$
x / S^{\{y\}} / F^{\{z\}} \rightarrow \begin{cases}1 / S^{\{*\}} / F^{\{*\}}, & x=1, \\ 2 / S^{\{*\}} / F^{\{*\}}, & x=2, \\ 4 / S^{\{*\}} / F^{\{*\}}, & x=4\end{cases}
$$

where

$$
S^{\{*\}}=\left\{S^{+}, S^{\prime}, S^{\infty}\right\}
$$

The solutions are equivalent with 1-1-3, 1-2-1, 1-2-2 and 2-2-2 within 76 Inventive Standard Solutions [6]-[8].

For instant, the specific framework for enhanced user experiences has been proposed by using the Standard Solution 1-2-2 [10]-[11]. This problem is typical Type-2 problem in Su-Field notations because it have solved by removing the harmful function [11]. Su-Field model $2 / S^{+} / F$ is the solution of this problem by using Su-Field notation. In other words, the Standard Solution 1-2-2 can be noted as $2 / S^{+} / F$ in Su-Field notations [11].

\section{THE CASES OF USER EXPERIENCE DESIGN ON GENERAL FRAMEWORKS}

The cases are the examples of enhancing the user experiences. The framework can generate the concept design for the various Information Technology applications. This session provides three cases of User Experience design for smartphone devices.

\section{A. Case 1: Enhanced LBS UX Design}

Location Based Service (LBS) is an information and entertainment service, accessible with mobile devices through the mobile network and utilizing the ability to make use of the geographical position of the mobile device by using Global Positioning System (GPS) [12]. GPS is a mandatory technology for LBS applications but it takes more than ten minutes to find the initial location position of a device. Assisted GPS (A-GPS) is design for gathering the initial position much faster but A-GPS is required higher application chipset process power. Currently, a LBS application is very common and it is embedded even in a low tier devices. The initial GPS position must be calculated before launching the LBS applications but required the additional process power.

Based on Su-Field model, standard solution 1-1-3 is suitable for calculating the initial location position of the device without extra resources [13] (see Fig. 2).

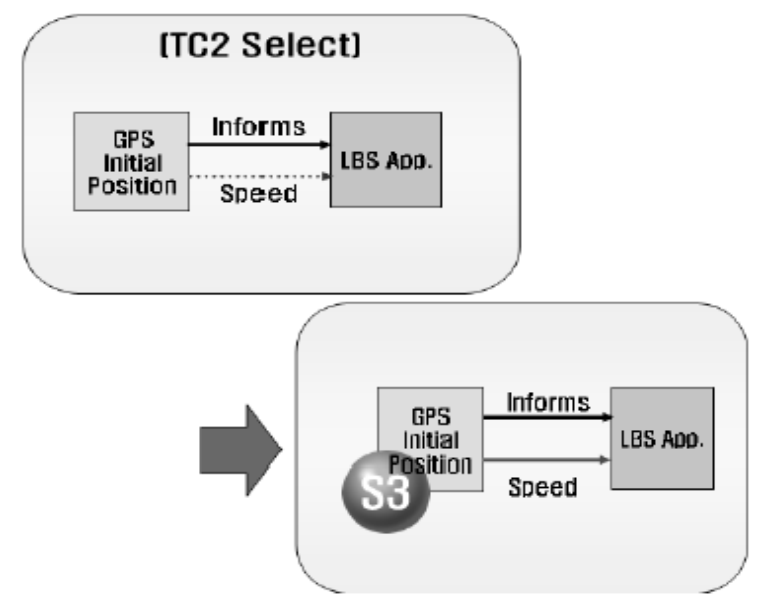

Fig. 2. Su-field model for UX of LBS application.

\section{B. Case 2: Enhanced WiFi UX Design}

Wireless Fidelity (Wi-Fi) allows electronic devices to exchange data wirelessly over a computer network. Wi-Fi suggests Wireless Fidelity, resembling the long-established audio-equipment classification term Hi-Fi. A device enabled with Wi-Fi, such as a personal computer, video game console, smartphone, tablet, or digital audio player, can connect to a network resource such as the Internet via a wireless network access points. Currently, most of mobile phones are Wi-Fi enabled for offloading their mobile networks. This technology becomes a major trend in IT industries because of the worldwide certification program by Wi-Fi Alliance [14]-[16].

The usage of Wi-Fi gets more popular in mobile industry. Wi-Fi capability is a common feature even in low tier devices. But the UX procedure of Wi-Fi setting is still focused on PC. Even though the Wi-Fi network is connected, the further procedures for authentication are needed and usual behavior is that a web browser is started. The authentication procedure on the browser such as ID and password is typical because it 
is typical procedure for PC.

The problem for enhancing the usage of Wi-Fi setting user experiences can be described as Su-Field models. Object $\left(S_{I}\right)$ is the user who wants to use Wi-Fi network that is a Hotspot near by a user and Tool $\left(S_{2}\right)$ is the user experiences (UX) for Wi-Fi setting on the device. Based on Su-Field Model, Problem Type-1-2 [17] as Su-Field Notation is the problem that contains the harmful action and the candidate solution is basically removing the harmful function:

From Fig. 1, the candidate solution of Problem Type 1-2 can be determined as follow [17]:

$$
1 / S^{\{-1\}} / F^{\{-1\}} \rightarrow\left\{\begin{array}{cc}
1 / S / F, & \because \text { Type }-1 \\
1 / S^{*} / F, & \left\{S^{*} \mid S^{\prime}, S^{+}, S^{2}, S^{\infty}, S^{n}\right\} \\
1 / S / F^{*}, & \left\{F^{*} \mid F^{\prime}, F^{\prime \prime}, F^{+}, F^{\infty}\right\} \\
1 / S^{*} / F^{*}, &
\end{array}\right.
$$

According to (2), the concept solution for enhanced software architecture is

$$
1 / S / F\{/ O\} \rightarrow 1 / S^{+} / F
$$

The concept solution (3) means that the UX has been revised to add additional software module for enhancing the LBS performance with minimal changing of the current user experiences [17].

\section{Case 3: MP3 Player List UX Enhancement}

The playlist in a MP3 player and a mobile phone is a basic user interface and recently user behavior has been changed because of memory expansion. Most of recent MP3 users can contain more than thousands of songs in one device and it is big changes when we compare with the situation of couple of years ago. Listing within thousand songs is heavy task these days.

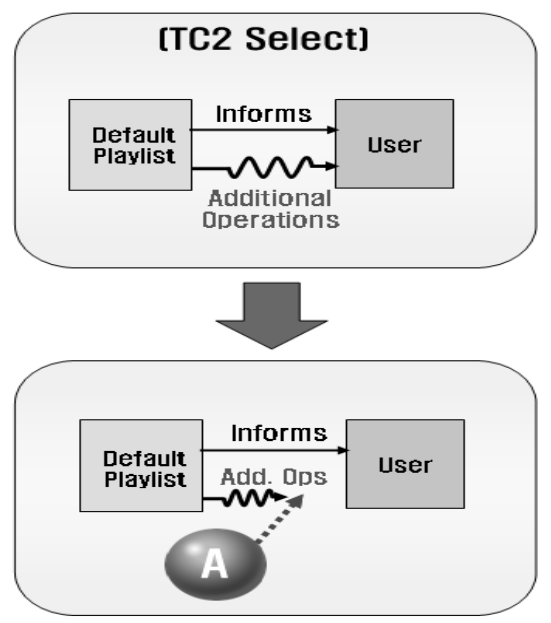

Fig. 3. Su-field model for UX enhancement.

There is another UX framework application to design for building the enhanced playlist. Substance-Field (Su-Field) model is for analyze the problems related existing technological systems [6]. Su-Field model analysis is applied for making innovative problems to the form of analysis model related to the current technical system. It means that Su-Field model is the tool for identifying problems in a technical system and finding innovative solutions to these identified problems [6]-[9]. Genrich Altshuller and his colleagues identified 76 standard solutions to fixing problematic Su-Field models based on their intensive research of a huge number of patents [4].

\section{CONCLUSION}

This research is targeting for developing the general framework of experience design to give user the easy way for operating the devices. Systematic Innovation methodology makes possible to design the new types of user experiences implementations. Even though the research is dedicated with mobile industries, the pattern of systematic approaches in the paper can be also applied to other industries.

\section{REFERENCES}

[1] J. W. Polderman and J. C. Willems, Introduction to Mathematical Systems Theory, a Behavioral Approach, Springer, New York, NY, 1998.

[2] R. H. Thaler and C. R. Simstein, "Improving Decision about Health, Wealth and Happiness," Penguin Books, New York, NY, 2008.

[3] Vincente and J. Kim, "the Human Factor: Revolutionizing the Way People Live with Technology," Routledge, New York, NY, 2006.

[4] G. Altshuller, And Suddenly the Inventor Appeared, TRIZ, the Theory of Inventive Problem Solving, Technical Innovation Center, Worecester, MA, 1996.

[5] X. Mao et al., "Generalized Solutions for Su-Field Analysis," The TRIZ Journal, August, 2007.

[6] J. Terninko, "Su-Field Analysis," The TRIZ Journal, February, 2000.

[7] J. Terninko, A. Zusman et al., Systematic Innovation: An Introduction to Theory of Inventing Problem Solving, CRC Press, Boca Raton, FL, 1998.

[8] K. Rantanen and E. Domb, Simplified TRIZ 1st ed., CRC Press, Boca Raton, FL, 2002.

[9] S. K. Kim, "Concept Design based on Substance-Field Model in Theory of Inventive Problem Solving," International Journal of Innovation, Management and Technology, vol. 3, no. 4, pp. 306-309, 2012.

[10] S. K. Kim, "Design of Event Driven DRM," IEEE Proceedings of IEEM, pp. 935-938, 2007.

[11] S. K. Kim, "Enhanced User Experience Design based on User Behavior Data by Using Theory of Inventive Problem Solving," IEEE Proceedings of IEEM, pp. 2076-2079, 2010.

[12] S. Wang, J. Min, and B. K. Yi, "LBS for Mobiles: Technologies and Standards," Proceedings of ICC, 2008.

[13] S. K. Kim, "Alternative Approach of Inventive Problem Solving for LBS Implementation," International Journal of Social Science and Humanity, vol. 1, no. 2, pp. 132-134, 2011.

[14] Wi-Fi Alliance. (2012). Wi-Fi Alliance Annual Report 2011. [Online] Available: http://www.wi-fi.org.

[15] Wi-Fi Alliance. (2012). Wi-Fi Alliance Roadmap. [Online]. Available: http:// www.wi-fi.org.

[16] Wi-Fi Alliance. (2012). Wi-Fi CERTIFIED Passpoint: A new program from the Wi-Fi Alliance to enable seamless Wi-Fi access in hotspots. [Online]. Available: http:// www.wi-fi.org.

[17] S. K. Kim, "Effective Wi-Fi Setting User Experience Design by Using Systematic Innovation Method," IEEE Proceedings of IEEM, Accepted, 2012.

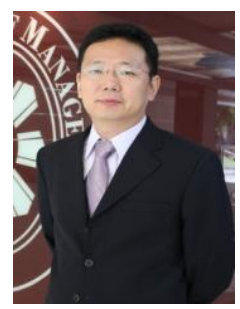

Song-Kyoo (Amang) Kim is an Associate Professor of Asian Institute of Management. He had been a technical manager and TRIZ specialist of mobile communication division at Samsung Electronics. He is involved in IT industries more than 10 years. Dr Kim has received his master degree of computer engineering on 1999 and Ph.D. of operations research on 2002 from Florida Institute of Technology. He is the author of more than 20 operations research papers focused on stochastic modeling, systematic innovations and patents. He had been the project leader of several 6 Sigma and TRIZ projects mainly focused on the mobile industry. 